\title{
AUGUSTE CHEZ DEUX AUTEURS DE L'ANTIQUITE TARDIVE, AUSONE ET MACROBE
}

Summary: Ausonius and Macrobius reflect both the positive image enjoyed by Augustus in the fourth century. They also illustrate the importance of Suetonius in Late Antiquity. The manner in which Ausone shows Augustus is not original. On the other hand Macrobius'Augustus, who is neither affected nor hieratic and supports mockery, implicitly contrasts by his conduct to the solemnity of the ceremony surrounding the emperors of the Lower Empire.

Key words: Augustus, Ausonius, Macrobius

On s'intéressera ici à deux auteurs de l'Antiquité tardive qui traitent d'Auguste selon des modalités et dans un dessein complètement différents, Ausone et Macrobe.

Les Caesares d'Ausone ${ }^{1}$ (c'est le titre habituel, en l'absence d'indication dans les manuscrits) se composent d'abord de trois séries de douze monostiques consacrées respectivement à l'ordre des douze premiers empereurs, à la durée de leur règne et à leur mort, puis d'une série de vingt-quatre tétrastiques sur les empereurs de César à Élagabal. Les monostiques sont écrits en hexamètres, les tétrastiques en distiques élégiaques. Chacune des deux parties est précédée d'une préface en vers : la première est une dédicace d'Ausone à son fils Hesperius.

Les Caesares ont peu retenu l'attention de la critique, si ce n'est pour les trois problèmes suivants ${ }^{2}$. Le dernier tétrastique ne comporte qu'un distique : l'œuvre s'arrêtait-elle à Élagabal ? C'est une fin logique sur la base des séries de douze, mais peu significative du point de vue historique (on attendrait un arrêt après Sévère Alexandre, dernier empereur de la dynastie).

\footnotetext{
${ }^{1}$ Toutes nos références sont données d'après The Works of Ausonius. Edition with Introduction and Commentary by R. P. H. GREEN. Oxford 1991.

${ }^{2}$ Voir GREEN, R. P. H. : Marius Maximus and Ausonius' Caesares. The Classical Quarterly 31 (1981) 223-236; GREen, R. P. H. : Ausonius' Fasti and Caesares revisited. The Classical Quarterly 49 (1999) 573-578.
} 
Ensuite, quel rapport entretiennent les Caesares avec l'œuvre donnée dans la liste des ouvrages d'Ausone dressée par Giovanni Mansionario (Jean de Matociis) vers $1320^{3}$ et ainsi décrite : item ad eundem de imperatoribus res nouas molitis a decio usque ad dioclenianum uersu iambico trimetro iuxta libros eusebij nannetici ystorici ? Les quatre vers qui introduisent les tétrastiques ${ }^{4}$ montrent qu'Ausone avait l'intention de faire suivre les monostiques des douze premiers empereurs d'une longue série. L'œuvre évoquée par Mansionario pourrait donc être soit la seconde partie des Caesares, soit un autre opuscule perdu, mais de toute façon sa description serait erronée (notamment pour la métrique, peut-être sous l'influence du Ludus septem sapientum, également un catalogue, qui lui est bien composé en trimètres iambiques ; quant à l'historien Eusèbe de Nantes, il résulte sans doute d'une confusion avec Eusèbe de Césarée).

Enfin, on a relevé quelques parallèles intrigants avec l'Histoire Auguste, qui ont amené à s'interroger sur une éventuelle source commune 5 .

Ces questions cependant n'importent pas pour notre sujet. La datation de l'œuvre est inconnue. L'insistance sur les mérites de l'adoption (à propos des Antonins) pourrait selon R. P. H. Green ${ }^{6}$ constituer un critère, mais il ne parvient pas à des conclusions claires.

Ausone suggère qu'il écrit de mémoire (préface des tétrastiques), mais en même temps déclare se fonder sur Suétone (préface des monostiques ${ }^{7}$ ), dont il atteste la notoriété au $\mathrm{IV}^{\mathrm{e}}$ siècle. Il est difficile d'identifier de manière certaine d'autres sources. Robert M. Sykes dans un $\operatorname{article~}^{8}$ et R. P. H. Green dans son édition notent quelques parallèles textuels, mais ils ne prouvent pas une influence.

L'œuvre ne se veut pas historique et le jugement porté sur chacun des empereurs est globalement conforme à la tradition. On n'a pas affaire non plus à un résumé ou une paraphrase de Suétone. Les Caesares doivent plutôt être rapprochés des Epitaphia heroum et ressortissent au genre de l'épigramme. Il s'agit, comme pour les épitaphes, de réduire le règne et la personnalité d'un empereur à quelques caractéristiques essentielles. Le but est donc la brièveté et la virtuosité, dans un art qui s'apparente à la poésie de la liste, chère à l'auteur.

Si Ausone dans les Caesares émet peu d'opinions personnelles, il privilégie néanmoins certaines thématiques : la durée longue ou brève des règnes (César, Auguste, Othon, Vitellius, Titus, Didius Julianus, Macrin), le mode de succession (les Antonins,

${ }^{3}$ Cette liste figure dans The Works of Ausonius, by GREEN (n. 1) 720.

${ }^{4}$ Nunc et praedictos et regni sorte sequentes expediam, series quos tenet imperii.

Incipiam ab Diuo percurramque ordine cunctos, noui Romanae quos memor historiae.

${ }^{5}$ Thomson, M. : Logodaedalia: Ausonius and the Historia Augusta. In Studies in Latin Literature and Roman History XIV. Ed. by C. DEROUX [Latomus n ${ }^{\circ} 315$ ], Bruxelles 2008, 445-475.

${ }^{6}$ Voir The Works of Ausonius, by GREEN (n. 1) 558.

${ }^{7}$ Préface des monostiques, vers 4-5 : quorum per plenam seriem Suetonius olim / nomina res gestas uitamque obitumque peregit.

${ }^{8}$ SYKES, R. M. : Some Observations on Ausonius' Caesars. Res publica litterarum XIII (1990) $271-277$. 
Caracalla), la différence entre le comportement de l'empereur avant et après son accession au pouvoir (Claude, Galba, Othon, Vespasien), la distinction entre bons et mauvais empereurs.

Parmi les procédés stylistiques auxquels il recourt notamment, on relèvera dans les tétrastiques : les symétries (Felix imperio, felix breuitate regni, vers 86 à propos de Titus) et les antithèses (quem fateare bonum, diffiteare parem, vers 101, pour Hadrien jugé inférieur à Trajan), qui s'appuient souvent sur des anaphores ou des polyptotes (sed iustior ordo est / complacuisse dehinc, displicuisse prius, vers 72-73, à propos de Galba qui plaisait avant d'être empereur mais a déplu une fois au pouvoir, alors que l'inverse aurait été préférable ; quia praemia regni / saepe indignus adit, non nisi dignus habet, vers 80-81, réflexion générale à propos du court règne de Vitellius) ; les pointes paradoxales (non faciendo nocens sed patiendo fuit, vers 65 pour Claude; hoc solum fecit nobile, quod periit, vers 77 pour Othon; hoc solo patriae, quod genuit, nocuit, vers 113 pour Marc Aurèle père de Commode); les jeux de sonorités (castrensis caligae cognomine Caesar, vers 58 pour Caligula; successit saeuo saeuior ingenio, vers 59 pour Caligula encore). Ausone cherche aussi à varier dans la présentation de chaque empereur. Par exemple, alors qu'il est généralement question des empereurs à la troisième personne, l'auteur s'adresse à Galba, Vitellius, Titus, Caracalla et Élagabal à la deuxième personne. Ou bien, arrivant à Néron, il refuse de rapporter ses crimes et renvoie à Suétone. Ou encore, s'agissant de Domitien, il se contente de déplorer que le troisième des Flaviens ait anéanti les bienfaits des deux premiers.

Le discours tenu sur Auguste n'est pas original, on l'a dit. Les monostiques insistent sur la longueur de son règne (Augustus post lustra decem sex prorogat annos, vers 19; Addidit Augustum diuis matura senectus, vers 31). Les tétrastiques qui lui sont consacrés reprennent la même idée, en soulignant que cela le fit regarder comme un dieu sur terre (Longaeua et numquam dubiis uiolata potestas / in terris positum prodidit esse deum, vers 52-53).

Il n'est pas autrement question d'Auguste chez Ausone.

Macrobe offre beaucoup plus de matière pour notre sujet. Au livre II des Saturnales, Aviénus, qui semble être le fabuliste, rapporte divers bons mots et anecdotes plaisantes : le chapitre 4 est consacré à Auguste, le 5 à sa fille Julie ${ }^{9}$. Auguste, nous dit Avienus (II 4. 1), eut toujours une propension pour les mots d'esprit, sans porter atteinte à son rang ni tomber dans l'impudeur ou la bouffonnerie. Ces anecdotes et bons mots, au-delà de leur caractère amusant, ont une visée plus haute : ils dessinent en creux un portrait moral de l'empereur.

Le chapitre 4 est divisé en deux parties : les bons mots que profère Auguste (II 4. 2-18), puis ceux dont il est victime (II 4. 19-31). Il n'y a pas de principe d'organisation net à l'intérieur de chacune des deux parties, même si on distingue des séries (par exemple II 4. 8-10 reposent sur des jeux de mots).

\footnotetext{
${ }^{9}$ Il n'existe pas d'étude spécifique sur le chapitre 4 ; sur le chapitre 5, on verra GRENADE, P. : Analyse d'un texte des Saturnales de Macrobe, Sat. II 5. 2-5. Revue des Études latines 32 (1954) 42-44; LoNG, J. : Julia-Jokes at Macrobius' Saturnalia: Subversive Decorum in Late Antique Reception of Augustan Political Humor. International Journal of the Classical Tradition 6.3 (1999-2000) 337-355.
} 
Dans la première catégorie (II 4. 2-18), il s'agit souvent de remettre à sa place un impertinent, un fâcheux ou un ridicule. Par exemple, Pacuvius Taurus réclame un don et affirme que le bruit s'est déjà répandu que l'empereur lui a accordé une grosse somme (II 4. 4). Pacuvius ajoute donc à sa demande un argument de contrainte : Auguste doit se mettre en conformité avec la rumeur qui anticipe la réalité. L'empereur répond à Pacuvius : « Mais toi, n'en crois rien ». Il refuse d'accorder le don, et fait comprendre à Pacuvius qu'il a conscience que la rumeur en question est fausse ou répandue par Pacuvius lui-même dans le seul but de le contraindre.

On a un schéma analogue dans l'anecdote qui suit immédiatement. Un commandant de cavalerie destitué réclame une gratification (II 4. 5), en expliquant qu'il la veut pour faire croire qu'il a obtenu d'Auguste une faveur et ainsi abandonné sa fonction. Il cherche donc à renverser la situation et à transformer une destitution en apparence de départ volontaire. Auguste répond : "Toi, affirme partout que tu l'as reçue, de mon côté je ne nierai pas te l'avoir donnée ». Auguste refuse la gratification réelle imméritée, mais accepte de se prêter à la fiction du départ volontaire.

Différente est l'anecdote sur Herennius (II 4. 6), également transmise par Quintilien (VI 3.64). Auguste avait ordonné à ce jeune homme adonné aux vices (deditum uitiis) de quitter le camp. Celui-ci le suppliait de revenir sur sa décision et lui adressait ces mots : « Que dirai-je à mon père? ». « Dis que je t'ai déplu » répond Auguste. La réplique, un peu leste, est à mettre en rapport avec les vices du jeune homme, c'est-à-dire l'homosexualité. Auguste fait comme si le jeune homme avait rejoint son camp seulement pour y satisfaire ses désirs homosexuels et que son unique critère de jugement était l'attrait physique. Il le rembarre ainsi et lui suggère en outre que quelqu'un dont les vices sont bien connus n'a pas à se soucier de maintenir les apparences avec son père.

Dans l'anecdote du soldat au visage défiguré par une blessure reçue au combat (II 4. 7), qui elle aussi se lit chez Quintilien (VI 3. 75 ; Quintilien attribue la réplique à César), la plaisanterie d'Auguste vise à rabattre la vantardise excessive du soldat. En lui disant : « Eh bien, quand tu prendras la fuite ne regarde jamais derrière toi », il fait comme si le soldat avait reçu sa blessure en fuyant, à un moment où il tournait la tête en arrière.

Dans l'anecdote sur Galba (II 4. 8), celui-ci se montre simplement maladroit. Galba donc, qui était bossu, plaidait une cause devant Auguste et répétait : " Ce que tu trouves incorrect, redresse-le (Corrige in me si quid reprehendis) ». Auguste lui dit : «Moi je puis te faire des observations (monere), mais te redresser (corrigere), je ne le puis », en jouant sur le sens du verbe corrigere.

Il en va de même du timide qui, tendant à Auguste un placet, tantôt avançait la main et tantôt la reculait, et à qui l'empereur dit : «Crois-tu donner une pièce de monnaie à un éléphant?» (II 4. 3 ; l'anecdote est aussi chez Quintilien VI 3. 59, et Suétone, Vies des douze Césars, «Auguste» 53. 5). Pline l'Ancien (VIII 14) rapporte que trois éléphants qui s'étaient pris d'affection pour des humains prenaient avec leur trompe les pièces de monnaie que les gens leur tendaient et les remettaient ensuite dans la poche (in sinum) des personnes qu'ils chérissaient. Peut-être l'habitude s'était- 
elle instaurée à Rome de tendre des pièces aux éléphants qui y arrivaient pour les jeux du cirque.

L'histoire du mauvais dîner (II 4. 13) illustre la patience avec laquelle Auguste supporte la grossièreté d'autrui. Auguste, invité à un dîner, se voit servir un repas frugal et sans aucune recherche (le texte ne précise pas si l'hôte agit ainsi par avarice ou désinvolture). En partant, il murmure (insusurrauit) simplement au maître de maison : «Je ne pensais pas être à ce point de tes intimes». En effet c'est seulement à des intimes qu'on peut servir un repas si simple. Auguste ne manifeste pas publiquement son mécontentement, il se contente de faire une remarque destinée à l'hôte seul (mais qui a finalement été connue d'un plus grand nombre, on ne sait par quel biais).

Par sa réplique au marchand, Auguste s'insurge contre la mauvaise qualité d'une pourpre qui lui a été vendue (II 4. 14). Comme il se plaignait du peu d'éclat d'une pourpre qu'il avait fait acheter, le vendeur lui conseille de la lever contre la lumière et de la regarder de bas en haut. «Eh quoi ? réplique Auguste. Pour que le peuple romain dise que je suis bien habillé, je devrai me promener sur une terrasse ? ».

Dans l'anecdote du nomenclateur (II 4. 15), Auguste montre de l'humeur devant les oublis répétés de celui-ci. On a ici le thème épigrammatique du professionnel incapable. Auguste donc déclare à son nomenclateur qui se rendait au forum : «Prends une lettre de recommandation, parce que là-bas tu ne connais personne ».

Dans la réplique à Vatinius (II 4. 16; elle figure également chez Quintilien VI 3. 77, qui la met dans la bouche de Cicéron), Auguste se moque de la prétention ridicule de celui-ci qui, malgré sa goutte, prétendait avoir fait une promenade de mille pas. C'est le thème du vieillard qui joue au jeune. Auguste réplique : « Je ne m'étonne pas; les jours sont un peu plus longs », comme si Vatinius avait eu besoin de toute la durée du jour pour parcourir cette distance.

L'éloge de Caton (II 4. 18), qui peut étonner puisque Auguste avait écrit un ouvrage contre lui (Suétone, Vies des douze Césars, «Auguste» 85. 1), a en fait plusieurs buts. Comme Auguste s'était rendu par hasard dans une maison où Caton avait habité, un certain Strabon, pour flatter l'empereur, critique l'obstination de Caton. Auguste déclare: "Tout homme qui ne veut pas bouleverser la forme présente de gouvernement est en même temps un bon citoyen et un homme de bien ». Par ces mots il trompe l'attente de Strabon et lui montre qu'il n'est pas dupe. En même temps il parle dans son intérêt propre en faisant, non sans quelque paradoxe, la critique des changements de régime et l'éloge du pouvoir en place. L'anecdote rappelle celle, rapportée par Plutarque (Vie de Cicéron 49), où Auguste, tombant par hasard sur un de ses petits-fils en train de lire Cicéron, fait, à la surprise de celui-ci, l'éloge de l'Arpinate. On est ici à la limite de la sententia politique ${ }^{10}$.

La lettre à Mécène (II 4.12) est d'une nature particulière, c'est une parodie, et de fait Suétone (Vies des douze Césars, «Auguste » 86. 3) nous apprend qu'Auguste, qui était dans l'écriture adepte de la simplicité, s'amusait à contrefaire le style de son ami pour s'en moquer. Ici, en une sorte d'oxymore, les biens précieux mentionnés

\footnotetext{
${ }^{10}$ Comme le note Golduust, B. : Rhétorique et poétique de Macrobe dans les Saturnales. Turnhout 2010,452 .
} 
(Vale, mel gentium, $\dagger$, ebur ex Etruria, lasar Arretinum, adamas supernas, Tiberinum margaritum, Cilniorum smaragde, iaspi figulorum, berylle Porsenae, carbunculum $\uparrow$ ), qui évoquent peut-être l'apogée de la civilisation étrusque, sont systématiquement associés à des régions d'où ils ne peuvent provenir, ainsi l'ivoire d'Étrurie, le diamant de l'Adriatique ou la perle du Tibre.

Dans d'autres cas, la réplique d'Auguste est un pur jeu de mots ou une plaisanterie, sans qu'il y ait de pointe contre une personne présente. Comme beaucoup de procès où l'orateur Cassius Severus était l'accusateur s'achevaient par un acquittement (cum multi Seuero Cassio accusante absoluerentur), et que parallèlement les travaux du forum d'Auguste traînaient en longueur, l'empereur déclara (II 4. 9) : « Je voudrais que Cassius mette aussi en accusation mon forum ». Il joue sur le sens du verbe absoluere, qui signifie à la fois «acquitter» et «achever». Car le forum alors serait, non acquitté, mais achevé.

De même, dans l'anecdote qui vient immédiatement après (II 4. 10), comme un certain Vettius avait labouré le terrain où était enseveli son père, ce qui est un acte impie, Auguste s'exclame : «Voilà qui est vraiment cultiver (colere) le souvenir de son père », en jouant sur les deux sens du verbe colere, « cultiver» et « honorer».

L'anecdote suivante est plus complexe (II 4. 11). Ayant appris que le roi Hérode avait fait tuer tous les enfants de moins de deux ans, dont son propre fils, Auguste s'écrie : "Il vaut mieux être le pourceau d'Hérode que son fils ». Cette déclaration repose sur le fait que les Juifs, s'abstenant de manger du porc, n'ont pas de raison de tuer cet animal. Auguste stigmatise à la fois la cruauté du roi et les règles alimentaires des Juifs, qui choquaient les Romains. C'est dans la littérature païenne la première allusion au massacre d'Hérode, et cela révèle sans doute une influence chrétienne sur Macrobe.

L'anecdote sur le matelas est une plaisanterie (II 4. 7). À la vente aux enchères des biens d'un chevalier qui avait vécu en dissimulant d'énormes dettes, Auguste achète son matelas, et donne comme raison : « Il doit favoriser le sommeil, le matelas sur lequel cet homme a pu dormir malgré le poids de ses dettes ». On sait par Suétone qu'Auguste avait besoin de beaucoup de sommeil (Vies des douze Césars, «Auguste » 78).

L'anecdote qui ouvre le chapitre (II 4.2) est un jeu de mots littéraire, déjà rapporté par Suétone (Vies des douze Césars, "Auguste » 85. 3). Auguste avait commencé une tragédie sur Ajax, et comme on lui demandait ce que devenait son Ajax, il répondit pour dire qu'il avait effacé ses vers : «Il s'est précipité sur une éponge », par allusion au suicide d'Ajax sur son épée.

La deuxième catégorie des bons mots du chapitre (II 4. 19-31) est celle des mots bons et railleries venant d'autrui et qu'Auguste a supportés sans s'irriter. Macrobe l'y déclare plus admirable que dans les plaisanteries dont il est l'auteur, car il sut « endurer avec égalité d'âme des mots qui allaient au-delà de la simple plaisanterie ( $m a-$ xime cum aequanimiter aliqua etiam iocis mordaciora pertulerit) » (II 4. 19; l'idée est répétée en II 4.25 et 26). 
Dans la grande majorité de ces anecdotes, soit le bon mot constitue une critique ou un reproche à Auguste, soit c'est le retournement d'une plaisanterie d'Auguste qui se trouve ainsi pris à son propre jeu.

La première anecdote (II 4. 20) est exemplaire de ce schéma de l'arroseur arrosé. Un provincial qui ressemblait de manière frappante à Auguste était venu à Rome. L'empereur le convoque, l'examine et lui demande : «Dis-moi, jeune homme, ta mère est-elle jamais venue à Rome ?»; il suggère ainsi que le provincial serait le fruit d'un adultère de sa mère avec son père à lui, Auguste. Le jeune homme répond par la négative et ajoute : "Mais mon père, lui, y est venu souvent ». Il renvoie l'insulte en suggérant qu'Auguste pourrait être né d'un adultère de sa mère, et que leur ressemblance s'expliquerait ainsi. Si Auguste a supporté d'entendre ce mot outrageant, c'est certainement parce qu'il a eu conscience d'être en tort et se voyait justement puni pour son indélicatesse.

Dans les autres cas la réplique est une critique plus ou moins explicite du comportement d'Auguste. Ainsi sous le triumvirat Pollion, contre qui Auguste avait composé des vers fescennins, déclare : «Moi, je me tais; car il n'est pas facile d'écrire (scribere) contre qui peut proscrire (proscribere) » (II 4. 21). C'est une claire attaque contre la toute-puissance d'Auguste triumvir, et Suétone souligne qu'il en a abusé (Vies des douze Césars, «Auguste » 27). Asinius Pollion était partisan d'Antoine, et son indépendance d'esprit est bien connue.

L'anecdote avec l'affranchi Licinius (II 4. 24) est différente. Cet affranchi avançait des sommes d'argent à Auguste quand il entreprenait des travaux. Or, un jour, Auguste falsifie le bon rédigé par l'affranchi en indiquant une somme plus importante. L'affranchi ne manifeste rien mais, à l'occasion de nouveaux travaux, écrit sur le bon : « Je mets à ta disposition, maître, la somme que tu voudras ». C'est une manière de dire qu'il n'a pas besoin de préciser la somme puisque Auguste la modifie. Par là il reproche à l'empereur à la fois sa malhonnêteté (falsifier un document) et son absence de courage (ne pas oser demander directement).

L'histoire avec le simple soldat (II 4. 26) repose aussi sur un reproche. Dans une maison de campagne, Auguste était dérangé la nuit par les cris d'un hibou. Il ordonne qu'on capture l'animal. Un soldat y parvient. Auguste lui donne mille sesterces en récompense. Le soldat déclare : "Je préfère qu'il vive », et relâche l'oiseau. Il jugeait évidemment la somme de mille sesterces trop modique.

Même type de reproche de la part du marchand d'esclaves Toranius (II 4. 28). $\mathrm{Au}$ cours d'un repas, Auguste apprécie les musiciens du marchand d'esclaves Toranius et leur fait donner du blé, alors que pour d'autres concerts il s'était montré généreux en argent. À un autre repas, plus tard, il réclame les mêmes musiciens et Toranius, pour refuser, avance l'excuse suivante : «Ils sont à la meule». Il veut dire par là que le faible prix dont Auguste a gratifié leur prestation précédente ne justifie pas qu'il les dérange de leur travail à la campagne. En même temps la meule, qui permet de moudre la farine, renvoie au blé donné par Auguste. Les esclaves seront plus productifs en actionnant la meule qu'en jouant de la musique, puisque Auguste leur accorde une si faible récompense. 
L'anecdote du vétéran qui demande assistance (II 4. 27 ; on la trouve aussi, avec des différences mineures, chez Dion Cassius LV 4) comporte également un reproche, mais il n'est pas d'ordre financier. Un vétéran en difficulté dans un procès demande à Auguste son aide. L'empereur lui fournit un avocat de sa suite. Le soldat lui crie alors en montrant ses blessures : "Mais moi, César, quand tu étais en danger lors de la bataille d'Actium je n'ai pas cherché un suppléant, mais j'ai combattu pour toi en personne ». Auguste alors vient le soutenir lui-même au tribunal, pour ne pas paraître orgueilleux ni ingrat.

C'est un reproche d'une autre nature qu'on trouve dans l'histoire du chevalier (II 4. 25). En tant que censeur, Auguste blâme à deux reprises un chevalier, mais à chaque fois sans fondement. Le chevalier, après s'être justifié, ajoute : «Dorénavant, César, quand tu enquêtes sur des personnes honorables, confie l'enquête à des personnes honorables ». Il souligne ainsi lourdement la mauvaise information d'Auguste.

Dans la majorité de ces anecdotes où il supporte une repartie cinglante, Auguste semble donc à quelque titre en faute, même si la réplique qu'il s'attire est parfois disproportionnée ou fait fi de son statut de premier personnage de l'État. Cependant dans deux cas il est la victime patiente de la grossièreté d'autrui. Un chevalier habitué au luxe, à qui dans un banquet offert par Auguste avait été servie une maigre grive, demande s'il peut la renvoyer (mittere). Auguste ne s'y opposant pas, il envoie (misit) la grive par la fenêtre (II 4. 22). Ici il s'agit moins d'une repartie que d'un comportement impoli : en outre, le chevalier joue sur l'ambiguïté du verbe mittere pour obtenir par une ruse hypocrite l'accord d'Auguste.

Le second cas est celui d'un sénateur dont Auguste rembourse intégralement les dettes et qui pour tout remerciement écrit à Auguste : « Et rien pour moi » (II 4. 23).

Les trois anecdotes qui terminent le chapitre ne rapportent pas à proprement parler des railleries que dut supporter Auguste, ce sont plutôt des situations où s'illustrent sa clémence et sa bonhomie. La première anecdote (II 4. 29) est celle, célèbre, des deux corbeaux qu'un homme a dressés à dire, l'un «Salut, César, vainqueur, imperator », l'autre « Salut, Antoine, vainqueur, imperator », pour pouvoir féliciter ainsi le vainqueur d'Actium, quel qu'il soit. Auguste ne s'irrite pas quand il découvre la rouerie.

La deuxième, de contenu assez proche, traite d'un pauvre cordonnier qui a dressé difficilement un corbeau à saluer l'empereur (II 4. 30). Comme celui-ci juge qu'il a assez d'oiseaux complimenteurs chez lui et refuse d'en acheter un de plus, l'animal ajoute la formule qu'il entendait répéter à son maître : «J'ai perdu ma peine et mon argent ». Auguste éclate de rire et acquiert l'oiseau à un prix élevé.

La troisième enfin (II 4. 31) est l'histoire d'un petit Grec qui, lorsque César descendait du Palatin, cherchait toujours à lui offrir une épigramme composée en son honneur. Un jour Auguste, voyant qu'il allait recommencer, trace sur une feuille une épigramme en $\operatorname{grec}^{11}$ et la fait passer au Grec qui allait l'aborder. Celui-ci la lit, la

${ }^{11}$ Selon Suétone, Vies des douze Césars, « Auguste » 89. 2, il ne parlait pas couramment le grec et ne se risquait pas à écrire dans cette langue. Cette affirmation un peu étonnante est cependant contredite par le texte même de Suétone, où l'on voit l'empereur improviser des vers grecs (98. 7). Peut-être y 
loue, met la main à sa pauvre bourse, en tire quelques pièces et dit en grec : «Par ta fortune, Auguste, si j'avais plus je te donnerais plus ». Tous les gens présents éclatent de rire et Auguste fait compter cent mille sesterces au pauvre Grec. L'anecdote s'apparente au schéma du retournement d'une plaisanterie dont nous avons parlé plus haut, mais ici la chose se fait sur le mode léger et amusant : Auguste, de manière facétieuse, inversait les rôles; on peut imaginer que son épigramme visait les solliciteurs importuns. Mais habilement le Grec tourne la situation à son avantage, en mimant le comportement qu'il voudrait que l'empereur adopte à son égard. Celui-ci, à la fois pour garder bonne figure et parce qu'il a apprécié l'humour du Grec, le récompense généreusement.

Le chapitre 5 sur les bons mots de Julie est, du point de vue qui nous occupe, intéressant par ce que Macrobe y dit d'Auguste à l'égard de sa fille. Selon Macrobe, Auguste se berçait d'illusions sur sa fille, la considérant comme une enfant gâtée (II 5. 4). Mais en réalité Auguste, s'il découvrit tard les vices de sa fille, selon Dion Cassius (LV 10), la relégua en 2 av. J.-C. dans l'île de Pandataria (aujourd'hui Ventotene, une des îles Pontines) ; il adoucit certes son sort quelques années plus tard en l'assignant à résidence à Rhegium (Reggio de Calabre), mais ne la rappela jamais auprès de lui (Suétone, Vies des douze Césars, « Auguste » 65 ; Tacite, Annales I 53. 1 ; Dion Cassius LV 10 et 13). Macrobe ne mentionne pas cette relégation, comme s'il ne voulait pas ternir l'image d'Auguste.

Les deux chapitres 4 et 5 s'inscrivent dans un ensemble où domine la gaieté (c'est la première moitié des conversations de l'après-midi du premier jour des Saturnales ; la seconde moitié est perdue). Macrobe y donne toujours la réplique spirituelle ou mordante (ou du moins sa partie essentielle) au style direct. Dans le chapitre 4 (le chapitre 5 fonctionne de manière un peu différente), il livre les anecdotes sans commentaire ou presque, ce qui les rend parfois difficiles à interpréter (ainsi pour II 4. 5 ; II 4. 6 ; II 4. 7). Les deux parties du chapitre montrent deux aspects d'Auguste: d'abord son goût pour les bons mots, ensuite sa capacité à supporter les railleries insultantes ou les insolences (que confirme Suétone, Vies des douze Césars, "Auguste » 54). On a vu que les bons mots d'Auguste visaient souvent à remettre des gens importuns ou ridicules à leur place, et que les bons mots contre Auguste contenaient généralement un reproche plus ou moins explicite et plus ou moins justifié. Globalement, la présentation qui est faite ici d'Auguste est favorable. Elle souligne sa bonhomie et sa patience. L'Auguste de Macrobe n'est ni hiératique ni affecté, il supporte la raillerie. Implicitement, ce comportement s'oppose à la solennité du cérémonial qui entoure les empereurs du Bas-Empire. Il ne faut pas voir là une critique de la part de Macrobe, mais peut-être un regret.

Ausone et Macrobe reflètent l'un et l'autre l'image positive dont jouissait Auguste $\mathrm{au} \mathrm{IV}^{\mathrm{e}}$ siècle et qu'on retrouve chez les abréviateurs (Aurelius Victor, pseudo-Aurelius Victor, Eutrope); la seule exception est Claudien, qui à deux reprises critique

a-t-il là une distinction entre contexte privé et situation publique, où l'empereur ne veut pas compromettre sa dignité en risquant de faire une faute. 
nettement Auguste en insistant sur sa férocité ${ }^{12}$. Ils témoignent aussi de l'importance de Suétone dans l'Antiquité tardive, qui est attestée également par l'anonyme Histoire Auguste. Suétone est explicitement la source d'Ausone. Pour Macrobe, la chose est moins claire. Il ne le mentionne jamais, et pourrait avoir utilisé un recueil de bons mots d'Auguste pour le chapitre II 4. On sait par les Disticha Catonis (qui ne remontent pas à Caton) que de tels recueils, sortes d'anas avant la lettre, existaient. C'est d'ailleurs ainsi que s'expliquent les divergences d'attribution de tel ou tel bon mot, les auteurs de ces recueils n'hésitant pas à prêter aux grands hommes des traits qu'ils n'avaient pas prononcés. On ne peut exclure non plus une tradition orale, malgré le temps écoulé depuis Auguste. Cependant, dans la mesure où il est banal dans l'Antiquité de ne pas nommer ses sources, nous inclinons à penser que Macrobe connaissait Suétone ${ }^{13}$.

Étienne Wolff

Université Paris Ouest Nanterre

adda-wolff@wanadoo.fr

ewolff@u-paris10.fr

${ }^{12}$ Guerre contre Gildon 49-51 (il a confisqué les droits des citoyens) ; Panégyrique pour le sixième consulat d'Honorius 116-118 (c'est par le massacre et en versant le sang qu'il a vengé son père).

${ }^{13}$ Voir aussi GuITTARD, CH. : Présence de Suétone chez un antiquaire et compilateur tardif : Macrobe et l'histoire du calendrier romain. In Présence de Suétone. Actes du colloque tenu à Clermond-Ferrand, 25-27 novembre 2004. Textes réunis par R. POIGNAULT. Clermont-Ferrand, Centre de recherches A. Piganiol 2009, 185-199 ; Flamant, J. : Macrobe et le néo-platonisme latin à la fin du IV siècle. Leiden 1977, 294-296, est plus réservé. 